\title{
Optimization of a light collection in the Shashlyk-type electromagnetic calorimeter with projective geometry for the NICA/MPD experiment.
}

\author{
Artur Durum ${ }^{1, *}$, Gennadiy Britvich ${ }^{1}$, Sergey Chernichenko ${ }^{1}$, Alexei Denisov ${ }^{1}$, Mikhail \\ Kostin $^{1}$, Yury Krechetov ${ }^{2}$, Andrei Yu. Semenov ${ }^{2}$, Alexander Sukhikh ${ }^{1}$, Nikolay Vlasov $^{2}$, and \\ Andrey Yanovich ${ }^{1}$ \\ ${ }^{1}$ NRC KI - IHEP, 142281, Protvino, Russia \\ 2 JINR, 141980, Dubna, Russia
}

\begin{abstract}
The MPD spectrometer at the NICA collider complex is currently under construction in Dubna. The main goal of the experiment is to obtain fundamental knowledge about the properties of hot and dense baryonic matter formed in heavy-ion collisions in the energy range of (411) $\mathrm{A}^{*} \mathrm{GeV}$. Crucial detector of the MPD experiment is a large-sized barrel electromagnetic calorimeter (ECal), which (together with the tracking system) will provide unique opportunities for the measurement and identification of a wide variety of charged and neutral particles carrying information about early stages of the interactions. Important tasks related to the construction of the Shashlyk-type MPD ECal are the development, production and study of the calorimeter modules with projective geometry. To improve performance of ECal, the light collection in the modules should be optimized. We present the methods and technologies developed to increase the light yield with different types and configurations of reflectors on the end of wavelengths shifting fibers. Expected characteristics of the calorimeter in detection of photons and electrons are presented and discussed.
\end{abstract}

\section{Introduction}

The Multi Purpose Detector (MPD) of the Nuclotron-based Ion Collider fAcility (NICA) in JINR (Dubna, Russia) is currently being created in order to obtain fundamental knowledge of the properties of hot and dense baryonic matter formed in heavy-ion collisions at a centre-of-mass energy range of 4-11 A* GeV and an average luminosity $10^{27} \mathrm{~cm}^{-2} \mathrm{~s}^{-1}$, where the critical point is suspected in the QCD phase diagram [1].

\footnotetext{
${ }^{*}$ Corresponding author: durum@ihep.ru
} 
Important detector in the new experimental setup is a large-sized barrel electromagnetic calorimeter (ECal) with projective geometry. The energy resolution of $\sim 5 \%$ for $1-\mathrm{GeV}$ photons and better than 1 -ns timing resolution are required to measure the spatial positions and energies of photons and electrons with high efficiency for MPD. Taking into account the requirements of high energy resolution, adequate timing resolution, dense active medium with the small Moliere radius, high segmentation of ECal, reasonable price, the Shashlyk-type electromagnetic calorimeter with projective geometry has been selected for the MPD/NICA [2]. It will consist of 38400 cells (towers) organized in 25 sectors; each tower has a sandwich structure of scintillator and lead plates with 16 wavelengths shifting (WLS) fibers that penetrate the plates to collect the scintillation light. The mechanical parameters of ECal tower are summarized in Table 1.

Table 1. Parameters of the ECal Shashlyk-type tower of MPD/NICA spectrometer. Possible changes are shown in parentheses.

\begin{tabular}{|l|l|}
\hline Transverse size, maximum & $40 \times 40 \mathrm{~mm}^{2}$ \\
Number of the layers & $220(210) \pm 1$ \\
Polystyrene scintillator, LEGO type & $1.5 \mathrm{~mm}$ thickness \\
Lead absorber & $0.3 \mathrm{~mm}$ thickness \\
Effective radiation length, $\mathrm{X}_{0}$ & $32.4 \mathrm{~mm}$ \\
Effective Moliere radius, $\mathrm{R}_{\mathrm{M}}$ & $62 \mathrm{~mm}$ \\
Radiation lengths, $\mathrm{Z}$ & $\sim 11.8 \mathrm{X}_{0}$ \\
Photodetector & HAMAMATSU S13360-6025PE MAPD (SiPM) \\
\hline
\end{tabular}

Often, the sampling factor (viz., a relation between radiation thickness of scintillator and lead plates) is considered to dominate in the energy resolution of a Shashlyk-type detector. But the combined contribution from photostatistics, light attenuation lengths of fibers and uniformity of light collections is comparable to the one from the sampling factor. According to KOPIO study [3] for Shashlyk-type tower prototype with 110x110 $\mathrm{mm}^{2}$ transverse size and $15.9 \mathrm{X}_{0}$ radiation length, the sampling-factor contribution is $2.69 \%$, and the energy-resolution contribution that corresponds to the light collection is $2.77 \%$. Therefore, the optimization of light collection in the tower gives us an opportunity to reach the better performance of ECal.

\section{WLS fibers and reflector type}

Impact electron or photon forms the electromagnetic shower in the ECal tower. The electrons and positrons pairs from this shower produce ultraviolet and blue light in the scintillator plate, which is absorbed by WLS fiber, and shifted to the green-light wavelength region. Then, this green light is transported to the photodetector. Light-trap efficiency, attenuation length of the fiber, the reflection coefficient of coating of the end of the fiber play a big role in the light collection.

We tested different types of fibers from Saint-Gobain [4] and Kuraray [5] companies with $1.0-\mathrm{mm}$ and $1.2-\mathrm{mm}$ diameters and single- or multi-cladding structure. For multicladding fiber, the light-trap efficiency is about $50 \%$ higher than for a single-cladding fiber. The single-clad fibers are faster (the decay time is $\sim 3 \mathrm{~ns}$ ) [4-6], but have worse radiationdamage resistance [7]. We used different types of coating of the far end of the fiber: Al Mylar, silver paint, Al mirror, a diffuse coating based on the $\mathrm{TiO}_{2}$ paint and epoxy; the $\mathrm{Al}$ Mylar was fixed on the fiber by the Scotch tape, and the Al mirror was made by aluminum sputtering. Before coating application on one end of the fiber, we polished both ends. To achieve a matte surface for a diffuse coating of the far end of the fiber, we used a fine-grain abrasive paper. For diffuse coating, we observed the big spot of light with high intensity 
from the fiber region that is close to the reflector (Fig. 1); it looks like that after reflection, the light direction was distributed in almost $2 \pi$ solid angle. In the similar experiment with an Al-mirror reflector, a smaller-sized light spot, and less intensity are observed, so the most of the reflected light went inside the solid angle of internal reflection, and only the small part of the light went out the fiber due to non-ideal Al mirror.
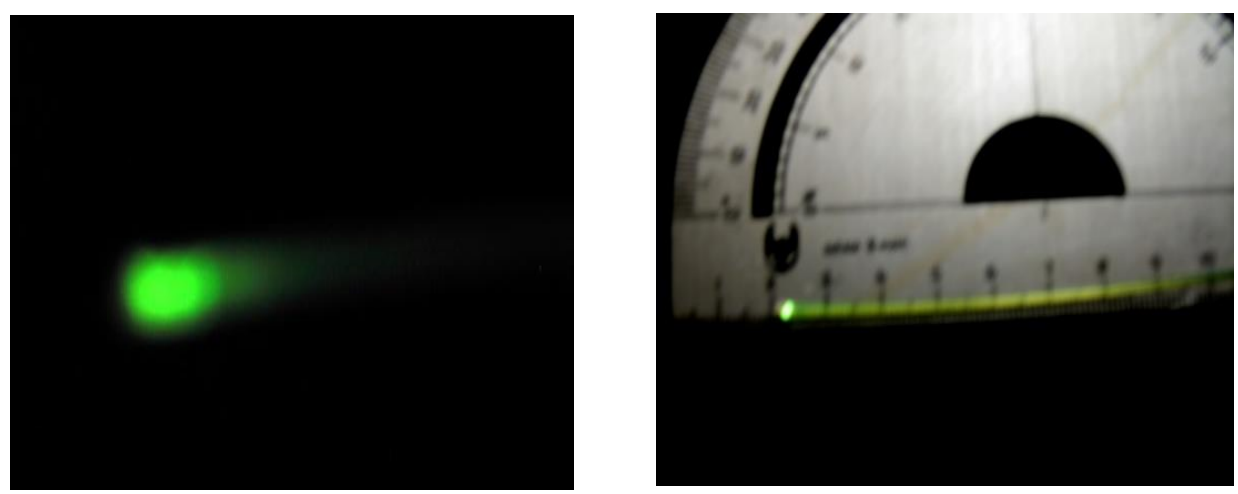

Fig. 1. Light output from side surface of fiber for diffuse coating. Pictures made in night shooting mode of camera.

Trying to return light back to the fiber and improve the situation for diffuse coating, we applied the coating for a side (cylindrical) surface of the fiber in addition to the end of fiber. The resulting configuration of the reflector looks like a "long boot" - the cylindrical reflective surface plus drop of coating. We called this configuration as "sapozhok" that is "boot" in Russian language. Due to the ECal/MPD tower design, the maximum height of "sapozhok" can not be more than $2 \mathrm{~mm}$.

During the production of tower prototypes, we measured the "sapozhok" height after the coating application, and the result was $1.7 \pm 0.35 \mathrm{~mm}$. Relatively big uncertainty is a result of a manual application of the coating. If "sapozhok" coating exceeds the desired size, the coating can be removed and applied again easily.

\section{Study of the reflectors on the end of WLS fibers}

To study reflectors, there is a method to measure direct light and the light that is reflected from the far end of the fiber in the same time; but this method requires very long fiber and a photodetector with extremely good timing resolution to separate the signals from the direct and reflected lights. We proposed an easier way of the measurement that includes a destruction of the measured fiber. The schematic view of experimental setup is shown in Fig. 2.

The light was injected to the WLS fiber from "Generic Ultra Bright Blue" LED with high temperature stability from RS Components Company [8]; this light has a peak emission wavelength of $470 \mathrm{~nm}$ and a typical opening angle of $15 \mathrm{deg}$. The fiber was rigidly attached to the PMT (FEU-84) window. The LED was easy to move along the test fiber in a black box. To monitor the setup stability, we used a pin photodiode. The signal from the PMT and amplified signal from the pin photodiode went to the LeCroy $2249 \mathrm{~W}$ QDC. The time of the measurement was about 45 minutes for one fiber. The LEDmonitoring system was excessive for a short measurement with one fiber, but it was absolutely needed for a long-time run.

The measurement consists of two steps: 
- We measured the light intensities with reflector (viz., mirrored "far-from-PMT" end of the fiber) with LED positioned on the fiber in the range of 10-140 cm from PMT with 5-cm step.

- Then the reflector was removed by cutting off the mirrored end of the fiber, and the fiber "far" end was placed inside an oil-filled black container and fixed in it with a plastic slab. This technique removes completely from the fiber the light that is emitted to the direction opposite to the photodetector, and simulates a "perfectly blackened" end of the fiber. Then the light-intensity measurements with non-mirrored fiber were repeated following the procedures from the step 1 .

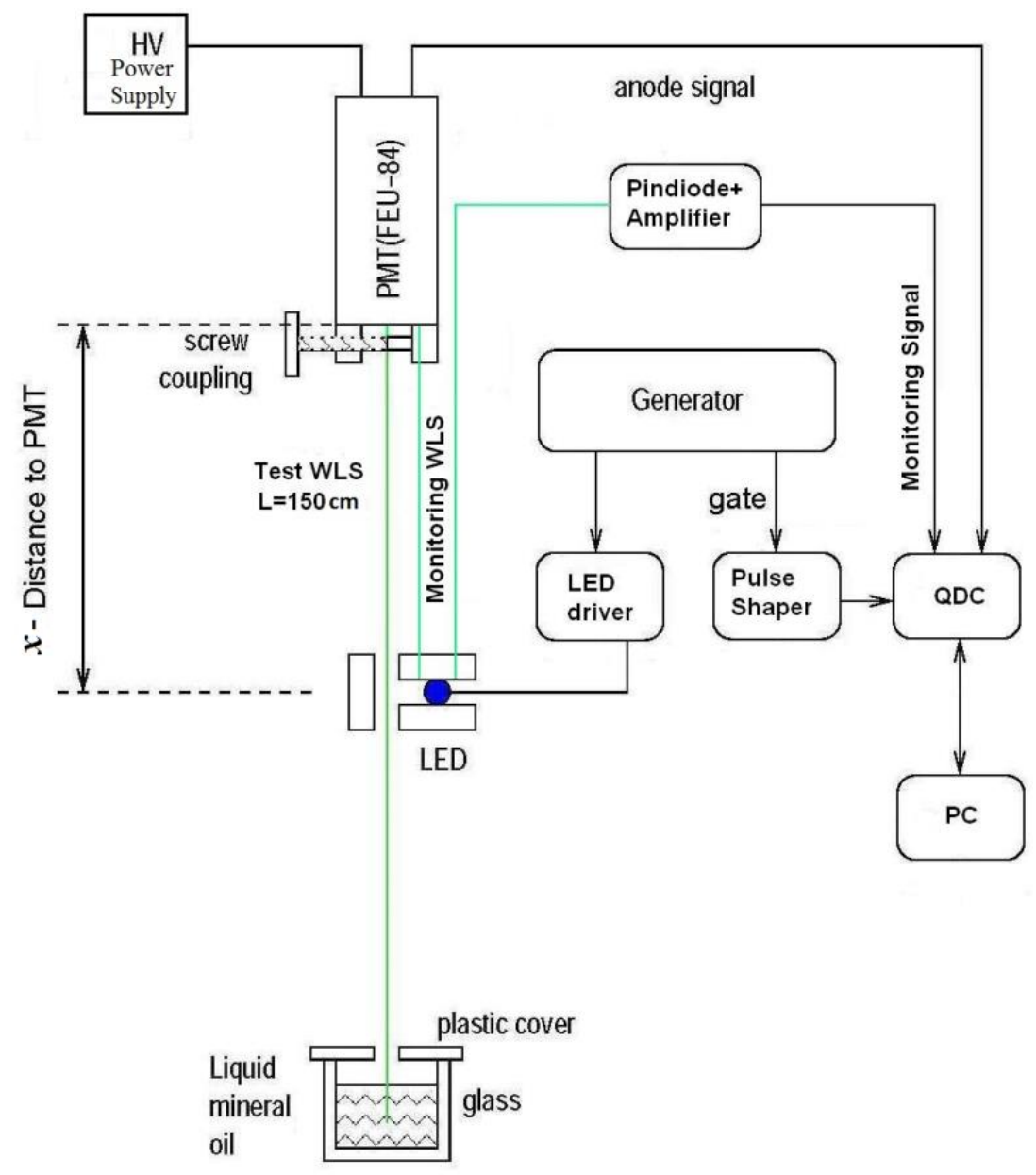

Fig. 2. Schematic view of the experimental setup to study the reflectors on the end of WLS fibers.

For each data point, we took 15000 LED events. Data were fitted to the Gaussian function. Mean and sigma parameters from the fit for each point in the 10-140 cm LEDposition interval were obtained, and pedestal subtraction was performed.

The dependence of the pedestal-subtracted mean light intensity as a function of the LED position relative to PMT for "non-mirrored" fiber was fitted to the following function:

$$
I_{\mathrm{NM}}(x)=\exp \left(\mathrm{p}_{1}+\mathrm{p}_{2 *} x\right)+\exp \left(\mathrm{p}_{3}+\mathrm{p}_{4 * x}\right),
$$


where $\lambda_{1}=-1 / \mathrm{p}_{2}$ is the attenuation length for the light traveled through external cladding (so-called "short exponent" in the attenuation), $\lambda_{2}=-1 / \mathrm{p}_{4}$ is the attenuation length for the light traveled inside the fiber core (so-called "long exponent" in the attenuation), $x-$ the distance between LED and PMT, $\mathrm{p}_{1}$ and $\mathrm{p}_{3}$ are normalization coefficients. The contribution from the cladding light is visible when the light from LED passes the fiber not-far-away from the photodetector (see Fig. 3), and part of reflected light is goes directly to the PMT. As one can see, $10 \%$ level of the cladding-light contribution to the total light yield was observed at $\sim 40 \mathrm{~cm}$ from PMT. The lengths of fibers in ECal towers are about $50 \mathrm{~cm}$. Therefore, we cannot ignore this cladding-light effect in our model to describe the light attenuation in the fiber.

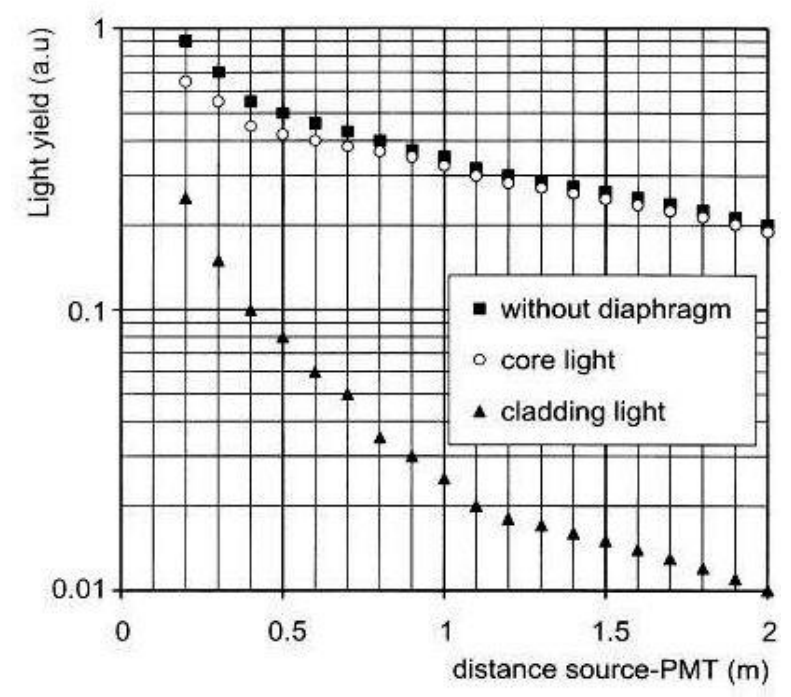

Fig. 3. Core and cladding light in PS/PMMA scintillating fiber [9].

The experimental data for mirrored fiber were fitted to the following function:

$$
I_{\mathrm{M}}\left(x, R_{\mathrm{j}}\right)=I_{\mathrm{NM}}(x)+R_{\mathrm{j}} * I_{\mathrm{NM}}(2 L-x),
$$

where $L=150 \mathrm{~cm}$ is the test-fiber length, and $R_{\mathrm{j}}$ is a parameter to take into account the effective reflection at the end of the fiber.

The experimental coefficient of effective reflection $R$ has been obtained from a minimum of $\chi^{2}$ criterion, which is defined with the expression:

$$
\chi_{\mathrm{j}}^{2}=\sum_{i=1}^{i=27}\left(\frac{I_{\mathrm{M}}\left(x_{\mathrm{i}}, R_{\mathrm{j}}\right)-I_{\mathrm{M}}\left(x_{\mathrm{i}}\right)}{\sigma\left(x_{\mathrm{i}}\right)}\right)^{2},
$$

where we took into account 27 experimental data points $I_{\mathrm{M}}\left(x_{\mathrm{i}}\right)$ with corresponding statistical errors $\sigma\left(x_{i}\right)$. The criterion was calculated for the $R_{\mathrm{j}}$ parameter in the interval of 0.1-1.0 with step of $\Delta R=0.01$; here 0.1 value for $R_{\mathrm{j}}$ corresponds to the situation of "almost no reflection", and 1 means "perfect reflection". The experimental data for Y11-200 MJ fiber of $1.2 \mathrm{~mm}$ in diameter with diffuse reflector type $\left(\mathrm{TiO}_{2}\right.$ plus epoxy) are shown on the fig. 4. Configuration of the reflector is the "sapozhok" for matte fiber end. 

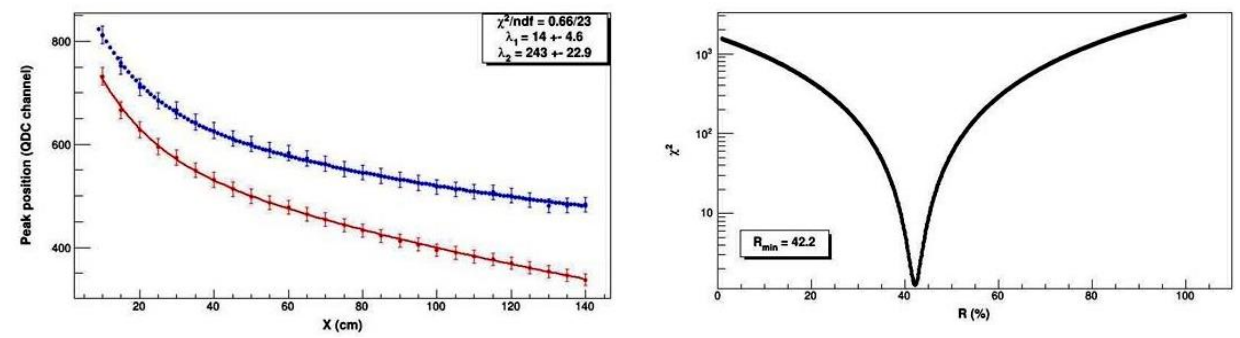

Fig. 4. Light-yield experimental data $I_{\mathrm{M}}$ for WLS fiber together with the $I_{\mathrm{NM}}$ dependence (Eq.2) are shown on the left panel as a function of the distance $x$ between light-injection point and the PMT. Blue points are experimental data for light yield with reflector, red points are experimental data for light yield without reflector. The data errors are dominated by systematic uncertainties. $\chi^{2}$ criterion from Eq. 3 is shown on the right panel as a function of the reflection parameter. The values of attenuation lengths obtained from the fit according to the Eq. 1 and the value of the effectivereflection coefficient $R$ for the minimum of $\chi^{2}$ criterion are shown.

The experimental results for the effective-reflection coefficient $R$ and expected ratios of the light yields from 50-cm-long fibers with reflectors in compare to these without reflectors are shown in Table 2. For each configuration and type of reflector, the values of the light yield in the presented ratios were averaged over the fiber length.

Table 2. The experimental results for the effective-reflection coefficient $R$ and expected ratios of the light yields from 50-cm-long fibers with reflectors in compare to these without reflectors.

\begin{tabular}{|l|c|c|}
\hline \multicolumn{1}{|c|}{$\begin{array}{c}\text { WLS types, types and configuration of } \\
\text { reflection }\end{array}$} & $\begin{array}{c}\text { Effective reflection } \\
\text { coefficient } R, \%\end{array}$ & $\begin{array}{c}I_{\mathrm{M}} / I_{\mathrm{NM}} \\
\text { for } L=50 \mathrm{~cm}\end{array}$ \\
\hline Y11-200, 1.2 mm, Al Mylar & $31.0 \pm 0.5$ & $1.13 \pm 0.01$ \\
\hline Y11-200, 1.2 mm, Silver paint (Al or Zn paint) & $23.4 \pm 1.4$ & $1.10 \pm 0.02$ \\
\hline $\begin{array}{l}\text { Y11-200, 1.2 mm, diffuse coating, polished } \\
\text { end }\end{array}$ & $31.0 \pm 0.8$ & $1.13 \pm 0.01$ \\
\hline $\begin{array}{l}\text { Y11-200, 1.2 mm, diffuse coating, polished } \\
\text { end, "sapozhok" < 2 mm }\end{array}$ & $42.8 \pm 1.5$ & $1.21 \pm 0.01$ \\
\hline $\begin{array}{l}\text { Y11-200, 1.2 mm, diffuse coating, matte end, } \\
\text { "sapozhok" }<2 \mathrm{~mm}\end{array}$ & $43.3 \pm 1.1$ & $1.20 \pm 0.01$ \\
\hline Y11-200, 1.0 mm, Al mirror, polished end & $82.2 \pm 0.7$ & $1.35 \pm 0.01$ \\
\hline Y11-200, 1.2 mm, Al mirror, polished end & $81.4 \pm 0.8$ & $1.38 \pm 0.01$ \\
\hline $\begin{array}{l}\text { Y11-200, 1.2 mm, Al mirror, polished end, } \\
\text { "sapozhok" 3.5 mm }\end{array}$ & $70.3 \pm 0.1$ & $1.30 \pm 0.01$ \\
\hline BCF-91A, $1.0 \mathrm{~mm}$, Al mirror, polished end & $81.5 \pm 0.6$ & $1.18 \pm 0.03$ \\
\hline BCF-91A, $1.2 \mathrm{~mm}$, Al mirror, polished end & $80.8 \pm 1.9$ & $1.24 \pm 0.03$ \\
\hline
\end{tabular}

From our data for light yields follows:

- the light yield for the 50-cm-long fiber of $1.2 \mathrm{~mm}$ in diameter with $\mathrm{Al}$ mirror is about 1.26 times higher than from the similar fiber of $1.0 \mathrm{~mm}$ in diameter;

- the light yield for the 50 -cm-long fiber of $1.2 \mathrm{~mm}$ in diameter with no mirror is about 1.28 times higher than from the similar fiber of $1.0 \mathrm{~mm}$ in diameter;

- the light yield for the 50 -cm-long fiber with $\mathrm{Al}$ mirror is about 1.23 times higher than from the similar fiber with diffuse coating; 
- usage of the "sapozhok" configuration for diffuse coating increases $R$ for about $12 \%$ that improves the light output for $6-7 \%$. But the same configuration for $\mathrm{Al}$ mirror has a negative effect on the light yields.

Our measurements on the muon beam for a tower with 16 Y11 fibers show that the light yield is about $1.16 \pm 0.03$ times higher if the $\mathrm{Al}$ mirror used in compare to the usage of $\mathrm{Al}$ Mylar; that is consistent with measurements with the single fiber and LED.

Systematic error associated with the optical re-connection of the fibers and the PMT window is estimated to be about $5 \%$. In this analysis, we did not study uncertainties that are connected with tolerance of diameter WLS fibers $<2 \%$ [5], the presence cracks on surface of fiber, quality of the fibers polishing and Al mirror, and statistical errors that are connected with an insufficient amount of fibers with each coating to measure. We estimate these uncertainties as $4 \%$ from the value or less. For example, for fiber with $\mathrm{Al}$ mirror the reflection coefficient depends on many factors like as coating technology, thickness of $\mathrm{Al}$ film, material purity, production time etc. The best-possible reflection coefficient for the perfect-quality $\mathrm{Al}$ mirror is $\sim 92 \%[10]$.

\section{Irradiation test}

Radiation hardness of scintillator and WLS fibers is well known [7]. But the radiation hardness of the diffuse coating $\left(\mathrm{TiO}_{2}\right.$ and epoxy) is unknown.

The radiation hardness requirements for ECal/PANDA experiment for proton-proton collision with average luminosity $\mathrm{L} \sim 2 * 10^{32} \mathrm{~cm}^{-2} \mathrm{~s}^{-1}$ is $10 \mathrm{G} /$ year, which is $10 \mathrm{kRad} /$ year. We took this value as a benchmark for ECal/MPD experiment which will work in the same energy range with average luminosity $\mathrm{L} \sim 2 * 10^{30} \mathrm{~cm}^{-2} \mathrm{~s}^{-1}$ for proton-proton collision. The irradiation was done at NRC KI - IHEP $\gamma$-source during 46.17 hours with total dose of 14.5 kRad. Three fibers were irradiated: with diffuse coating reflectors in "sapozhok" configuration on matte and polished end of fibers and without reflector as a reference. The measurements of light yield were done as described in Section 3. The results are shown in Table 4.

Table 4. The ratio of light yields before and after irradiation test for fibersY11-200. The result is shown for different fibers lengths of $50 \mathrm{~cm}, 120 \mathrm{~cm}$ and $140 \mathrm{~cm}$.

\begin{tabular}{|l|c|c|c|}
\hline Name & $\begin{array}{c}I_{0} / I_{\mathrm{rad}}, \\
L=50 \mathrm{~cm}\end{array}$ & $\begin{array}{c}I_{0} / I_{\mathrm{rad}}, \\
L=120 \mathrm{~cm}\end{array}$ & $\begin{array}{c}I_{0} / I_{\mathrm{rad}}, \\
L=140 \mathrm{~cm}\end{array}$ \\
\hline $\begin{array}{l}\text { Y11-200, } 1.2 \mathrm{~mm}, \text { diffuse coating, } \\
\text { matte end, "sapozhok" }<2 \mathrm{~mm}\end{array}$ & $1.06 \pm 0.03$ & $1.08 \pm 0.03$ & $1.09 \pm 0.03$ \\
\hline $\begin{array}{l}\text { Y11-200, } 1.2 \mathrm{~mm}, \text { diffuse coating, } \\
\text { polished end, "sapozhok" }<2 \mathrm{~mm}\end{array}$ & $1.06 \pm 0.03$ & $1.09 \pm 0.03$ & $1.10 \pm 0.03$ \\
\hline Y11-200, 1.2 mm, w/o reflector & $1.03 \pm 0.03$ & $1.07 \pm 0.03$ & $1.08 \pm 0.03$ \\
\hline
\end{tabular}

We did not see any influence of diffuse coating to radiation hardness of fibers.

\section{Conclusions}

Different types of the fibers were tested. The Kuraray Y11-200 multi-cladding fibers of $1.2 \mathrm{~mm}$ in diameter were selected by ECal/MPD management due to the best light yield and good decay time to improve photo-statistical contribution in the energy resolution. The problems because of too small bend diameter for this type of fibers which can occur during the mass production of ECal modules were solved into design. Slight increase of the non- 
uniformity contribution (in the total energy resolution) due to larger diameter of holes for fibers is not significant.

According to our research, the diffuse coating (based on the $\mathrm{TiO}_{2}$ paint and epoxy) in "sapozhok" configuration was selected as the reflector for the fiber ends by ECal/MPD management. It has the good effective reflection coefficient, good radiation hardness, does not need for high quality polishing of the fiber ends that means an easy and fast massproduction. It is not expensive reflector such as we plan to use the same glue-paint as a reflective and supporting material for the edges of scintillator plates in the module between towers. The negative aspects of this decision are the relatively small light output (in compare to the Al-mirrored fibers) and not-perfect uniformity of the light collection along the tower.

The reported study was supported by the Russian Foundation for Basic Research (RFBR), research project № 18-02-40083.

\section{References}

1. D. Blaschke et al., Eur. Phys. J. A 52, 267 (2016)

2. V. Golovatyuk et al., Eur. Phys. J. A 52, 212 (2016)

3. G.S. Atoyan, et al., Nucl. Instr. and Meth. A 320, 144 (1992)

4. Saint-Gobain Cristaux, 104 Route de Larchant, BP 521, 77794 Nemours CEDEX, France

5. Kuraray America, Inc., 200 Park Ave., NY 10166 USA

6. V. Brekhovskikh et al., CERN-LHCb-2000-039 (2000)

7. G. Britvich et al., CERN-LHCb-2000-037 (2000)

8. RS Components Stock Number: 235-9922

9. Ph. Rebourgeard et al., Nucl. Instr. and Meth. A 427, 543 (1999)

10. G. Hass, J.E. Waylonis, J. Opt. Soc. Amer., v. 51, №7, 719 (1961) 